\title{
ULTRASONOGRAPHY AND SURGERY OF CANINE BILIARY DISEASES
}

\author{
K. VÖRÖS ${ }^{1 *}$, T. NÉMETH ${ }^{2}$, T. VRABÉLY ${ }^{1 \S}$, F. MANCZUR $^{1}$, J. TÓTH $^{2 \#}$, Melinda MAGdus $^{1 \&}$ \\ and Edina PERGE ${ }^{3 \div}$ \\ ${ }^{1}$ Department and Clinic of Internal Medicine, ${ }^{2}$ Department of Surgery and Ophthalmology, \\ ${ }^{3}$ Department of Pathology and Forensic Medicine, Faculty of Veterinary Science, \\ Szent István University, H-1400 Budapest, P.O. Box 2, Hungary
}

(Received August 22, 2000; accepted October 30, 2000)

Findings of hepatic and gallbladder ultrasonography were analyzed in 12 dogs with gallbladder and/or extrahepatic biliary tract obstruction and compared with the results of exploratory laparotomy. Hepatic ultrasonography demonstrated normal liver in 2 dogs and hepatic abnormalities in 10 animals. The following ultrasonographic diagnoses were established compared to surgical findings: gallbladder obstruction caused by bile sludge (correct/incorrect: 1/2, surgical diagnosis: choleliths in one case), gallbladder obstruction caused by neoplasm $(0 / 1$, surgical diagnosis: mucocele), gallbladder and extrahepatic biliary tract obstruction due to choleliths $(3 / 3)$, extrahepatic biliary tract obstruction caused by pancreatic mass $(1 / 1)$ and small intestinal volvulus (1/1). Bile peritonitis caused by gallbladder rupture (4/4) was correctly diagnosed by ultrasound, aided with ultrasonographically-guided abdominocentesis and peritoneal fluid analysis. Rupture of the gallbladder should be suspected in the presence of a small, echogenic gallbladder or in the absence of the organ together with free abdominal fluid during ultrasonography. Laparotomy was correctly indicated by ultrasonography in all cases. However, the direct cause of obstruction could not be determined in 2 of the 12 dogs by ultrasonography alone.

Key words: Ultrasonography, surgery, dog, gallbladder, biliary tract

The incidence of the diseases of the gallbladder and the extrahepatic biliary tract is relatively low in the dog. These disorders usually demonstrate nonspecific clinical signs such as anorexia, icterus, vomiting, and abdominal pain or distension (Schall et al., 1973; Martin et al., 1986; Hager, 1989). The main goal of diagnosis is to differentiate between intrahepatic and extrahepatic diseases in order to determine prognosis and to select proper therapy. In this respect, bio-

\footnotetext{
* Corresponding author; E-mail: kvoros@univet.hu; Fax: +36 (1) 478-4137

$\S$ Present address: Echocard Diagnostic Centre, H-1135 Budapest, Lehel u. 43-47, Hungary

\# Present address: Hauptstrasse 18, 42579 Heiligenhaus, Germany

${ }^{\&}$ Present address: H-1124 Budapest, Fodor u. 52, Hungary

$\div$ Present address: Central Veterinary Institute, H-1149 Budapest, Tábornok u. 2, Hungary
} 
chemical examinations of the blood and urine, and plain film radiography often provide limited information. Cholecystography, cholangiography, and biliary scintigraphy require sophisticated equipment and are of limited use in the icteric animal, therefore these techniques are less frequently applied (Fossum and Willard, 1995).

Ultrasonography has been widely used to image the liver and gallbladder of the dog and cat, providing important information on both intra- and extrahepatic biliary disorders and on pathological processes of the surrounding abdominal organs (Nyland and Park, 1983; Vörös et al., 1991; Biller et al., 1992; Lamb, 1995; Nyland et al., 1995). In the dog, there are publications on the ultrasonographic appearance of cholelithiasis (Cartee, 1981; Kirpensteijn et al., 1993), cholecystitis (Lamb, 1990; Rivers et al., 1997), and gallbladder bile sludge (Gutbrod et al., 1987; Vörös et al., 1991). Thickening of the gallbladder wall in association with primary biliary adenocarcinoma in a dog has also been described (Brömel et al., 1998a). Sonographic signs of experimental bile duct ligation (Nyland and Gillett, 1982) and those of naturally occurring extrahepatic biliary obstruction have been reported (Diez-Bru et al., 1997). Ultrasonographicallyguided percutaneous cholecystocentesis has also been described as a diagnostic tool in canine cholecystitis (Rivers et al., 1997). Only one case report has been published on clinical ultrasonographic and surgical findings of gallbladder rupture due to cholecystitis and cholelithiasis in the dog (Brömel et al., 1998b).

The major goals of the present study were to demonstrate the applicability of ultrasonography in diagnosing gallbladder and extrahepatic biliary tract disorders and to assess the accuracy of this method in the indication of surgical treatment. Therefore, ultrasonographic findings of 12 dogs were compared with surgical findings obtained during laparotomy and cholecystectomy.

\section{Materials and methods}

The case records of dogs admitted to the Department of Internal Medicine within four years were reviewed. Twelve dogs which had been treated surgically for extrahepatic biliary tract disease were identified. The dogs included in the study weighed between 8 and $60 \mathrm{~kg}$ and had a mean age of 6.3 years (range 2 months to 12 years). The patients represented various breeds and there were 8 males and 4 females in the population. A detailed physical and laboratory examination including a complete blood count, determination of total and conjugated bilirubin concentration, aspartate aminotransferase (AST), alanine aminotransferase (ALT) and alkalic phosphatase (AP) activities, and routine urinalysis were performed in all cases. Abdominal radiography was done on 6 dogs to evaluate liver size, shape, gallbladder content (i.e. gas or radiopaque calculi), adjacent organs and the presence or absence of peritoneal fluid. 
Indications for hepatic and gallbladder ultrasonography included the presence of icterus, hepatomegaly, cranial or mid-abdominal mass, peritoneal effusion, hyperbilirubinaemia and elevated liver enzyme activities (Nyland and Park, 1983; Vörös, 1993; Nyland et al., 1995).

Abdominocentesis and analysis of the peritoneal fluid were performed in 4 dogs demonstrating physical signs and/or ultrasonographic findings of the presence of peritoneal effusion.

Ultrasonographic examinations were done with a Brüel and Kjaer 1846 ultrasound system, using 5.0, 6.0 or $7.0 \mathrm{MHz}$ real-time mechanical sector transducers (Brüel and Kjaer, Naerum, Denmark) depending on the size of the patients. Sagittal and transversal ultrasonographic planes were used for liver and gallbladder examinations and ultrasound images were analyzed as described previously (Nyland and Park, 1983; Vörös et al., 1991; Nyland et al., 1995). Ultrasonographic findings of the gallbladder (size, shape, wall, content) and the appearance of the biliary tract (intrahepatic and extrahepatic ducts) were evaluated to determine the presence and to reveal the cause of nonobstructive and obstructive biliary diseases.

Obstructive gallbladder or extrahepatic biliary tract diseases indicating surgery were diagnosed ultrasonographically when signs of dilatation of the extrahepatic biliary ducts (i.e. cystic and common duct) were present with or without dilated intrahepatic biliary vessels (Nyland and Gillett, 1982; Nyland et al., 1995), or when a large amount of biliary sludge and/or choleliths were seen within the highly dilated gallbladder, especially at the neck of the organ (Gutbrod et al., 1987; Kirpensteijn et al., 1993).

In addition, cranial intraabdominal organs (pancreas, gastrointestinal tract) were examined ultrasonographically and the presence or absence of free peritoneal fluid was recorded.

All dogs underwent exploratory laparotomy. Abdominal surgery was decided when ultrasonographic findings suggested obstructive disease of the gallbladder or the extrahepatic biliary system or when results of abdominocentesis revealed bile peritonitis. Anaesthesia was premedicated with diazepam, induced with thiopental and maintained with halothane and oxygen. During surgery, the gallbladder and the extrahepatic ducts of each dog were examined intraoperatively for evidence of intraluminal obstruction (caused by calculi or inspissated bile), visible signs of wall necrosis, acute or chronic rupture and the presence or absence of omental and hepatic adhesions as described previously (Church and Matthiesen, 1988). Antegrade cholecystectomy was performed according to earlier descriptions (Bjorling, 1975; Walshaw, 1985). In dogs with rupture of the gallbladder, massive abdominal lavage using warm 0.9 per cent saline solution was applied and a suction drain was placed in the cranial abdominal cavity of 4 dogs to provide local peritoneal drainage and to prevent local bile peritonitis. Isotonic fluids and cephalosporin antibiotics were given postoperatively to all dogs surviving surgery. Necropsy was performed in 5 cases, including liver and gallbladder histology of specimens stained with haematoxylin and eosin. 


\section{Results}

Signalment, ultrasonographic, surgical and postmortem findings are summarized in Table 1. The most common clinical findings indicating hepatic and/or gallbladder abnormalities included icterus, liver enlargement, cranial abdominal mass, abdominal pain and signs of abdominal free fluid accumulation. Hyperbilirubinaemia and elevated liver enzymes (AST, ALT, AP) of the serum were the most characteristic laboratory findings suggesting hepatic disorders.

Abdominal radiography of the 6 dogs demonstrated enlarged liver in 4 cases. In Case 11, radiographic signs of small intestinal volvulus were seen. Abdominocentesis of the 4 dogs revealed bile peritonitis in all of these patients on the basis of the fluid analysis (presence of macrophages, neutrophils, and a bilirubin concentration higher than that of the serum).

Ultrasonographic examination of the liver demonstrated normal liver in 2 dogs, and hepatic abnormalities in 10 dogs. Diffuse hepatic disease with increased echogenicity was diagnosed in 7 patients. A histological analysis of intraoperative liver biopsy samples or necropsies were done in 4 of these 7 animals and provided a diagnosis of hepatosis. A small, bright liver was seen in one case, suggesting liver cirrhosis, which was confirmed by necropsy. Multiplex hypoechoic focal alterations were detected in two dogs and hepatic neoplasms were suspected in these cases. Haemangiosarcoma of the liver was diagnosed by liver biopsy and hepatic necrosis was seen at necropsy in these 2 dogs, respectively.

Dilatation of the gallbladder was detected in 9 dogs. Small, poorly demarcated gallbladder was seen in two dogs and the gallbladder could not be identified in one dog. These three dogs had gallbladder wall perforation and bile peritonitis according to the findings of exploratory laparotomy and/or necropsy. Thickening of the gallbladder wall was revealed in 4 dogs and different amounts of gallbladder sludge (echogenic debris and inspissated bile) were seen in 8 dogs. It was not possible to identify the wall of the gallbladder in some cases, when the gallbladder was full of highly echogenic sludge. In one dog, a parenchymal mass similar to intraluminal neoplasm was suggested, but this alteration proved to be thickened, mucoid bile during cholecystectomy.

Gallbladder calculi with characteristic shadowing and gravitation (Fig. 1) were detected in 3 dogs, although gallbladder cholelithiasis was diagnosed surgically in 7 dogs. Of the remaining 4 dogs with cholelithiasis, only highly echogenic sludge was seen with gallbladder dilatation and wall thickening in two dogs and with a small, poorly demarcated gallbladder in two other dogs (Fig. 2). No ultrasonographic signs of gallbladder perforations (i.e. leaking of gallbladder content or discontinuity of the gallbladder wall) were detected in any of the dogs.

Dilatation of the cystic and common duct were seen in five dogs, in 3 of which the alteration proved to be obstructive cholelithiasis according to the surgical findings. In one dog, extrahepatic biliary tract obstruction was caused by pan- 
creatic disease (acute pancreatitis) and by small intestinal volvulus in another dog. These two diagnoses were suspected during abdominal ultrasonography and were confirmed by exploratory laparotomy.

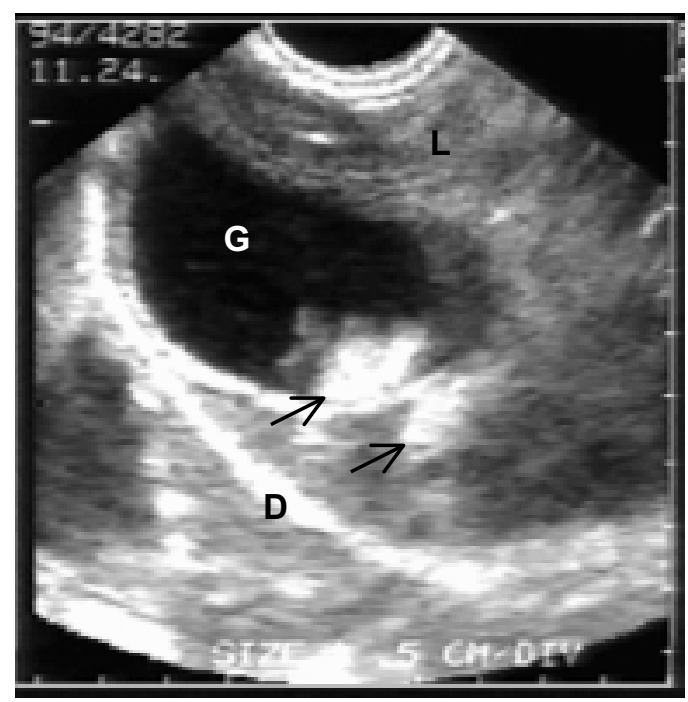

Fig. 1. Sagittal ultrasonographic image of a distended gallbladder with bile stones obstructing the cystic duct (arrow) in a dog (Case 4). D: diaphragm, L: liver, G: gallbladder

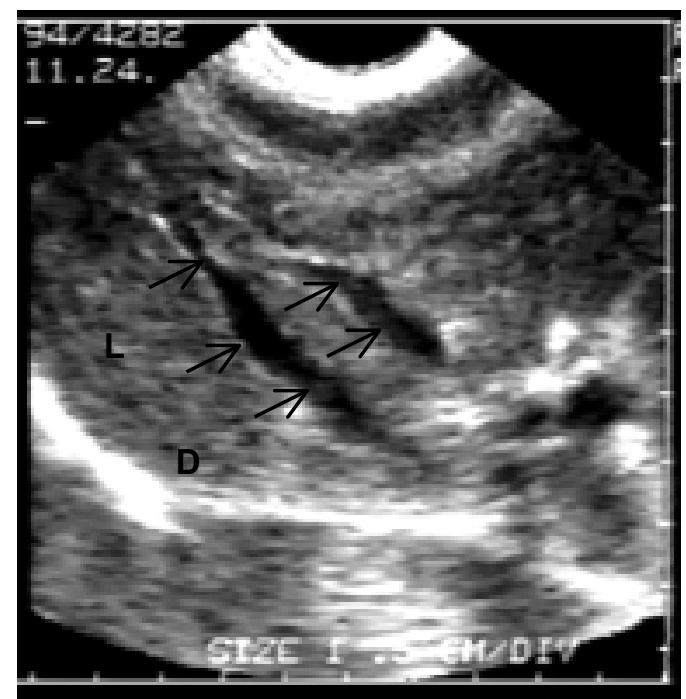

Fig. 2. Dilatation of the intrahepatic bile ducts in the same dog (Case 4) as in Fig. 1.

Bile ducts are marked with small arrows. D: diaphragm, L: liver 
VÖRÖS et al.

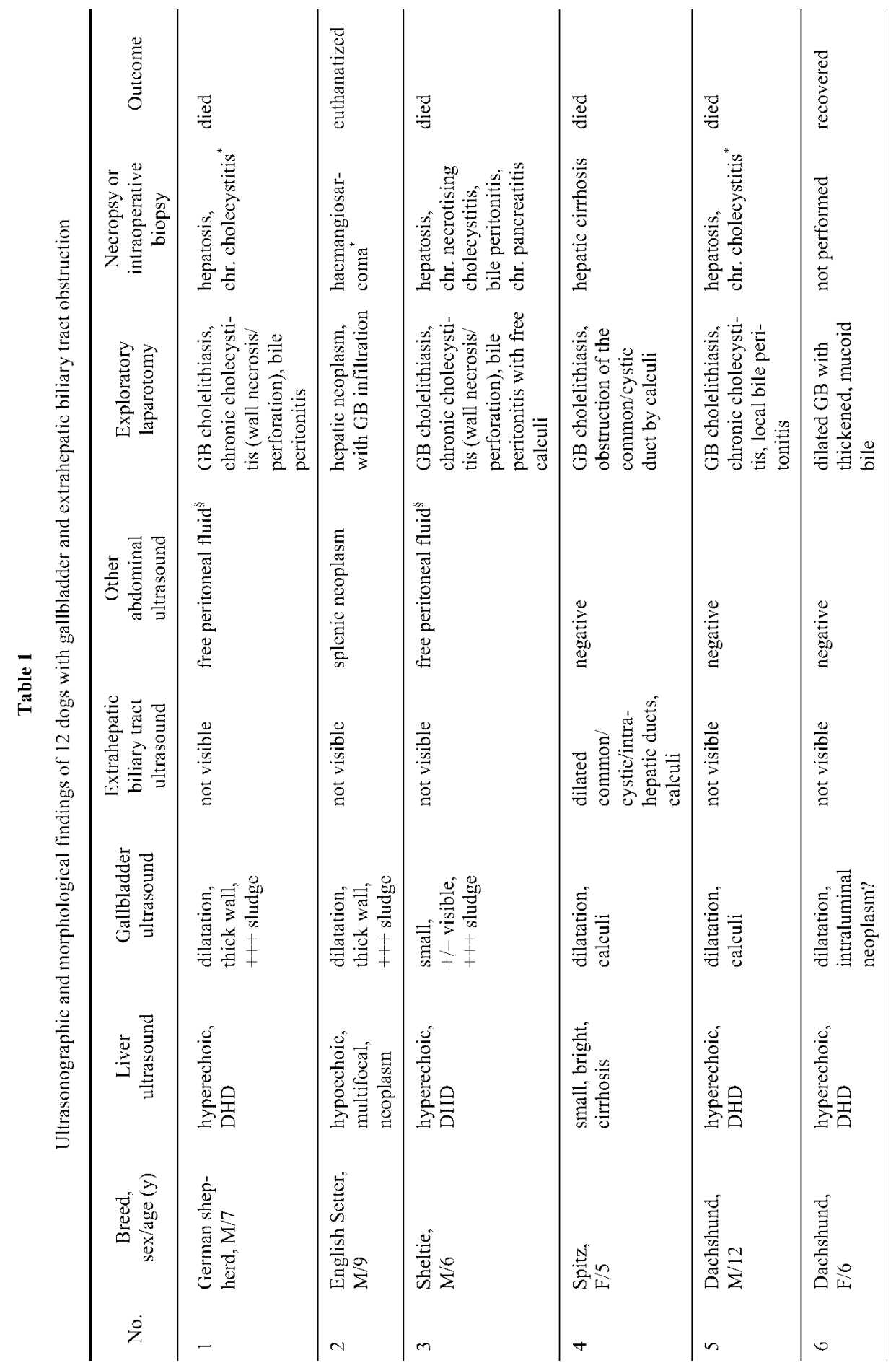

Acta Veterinaria Hungarica 49, 2001 


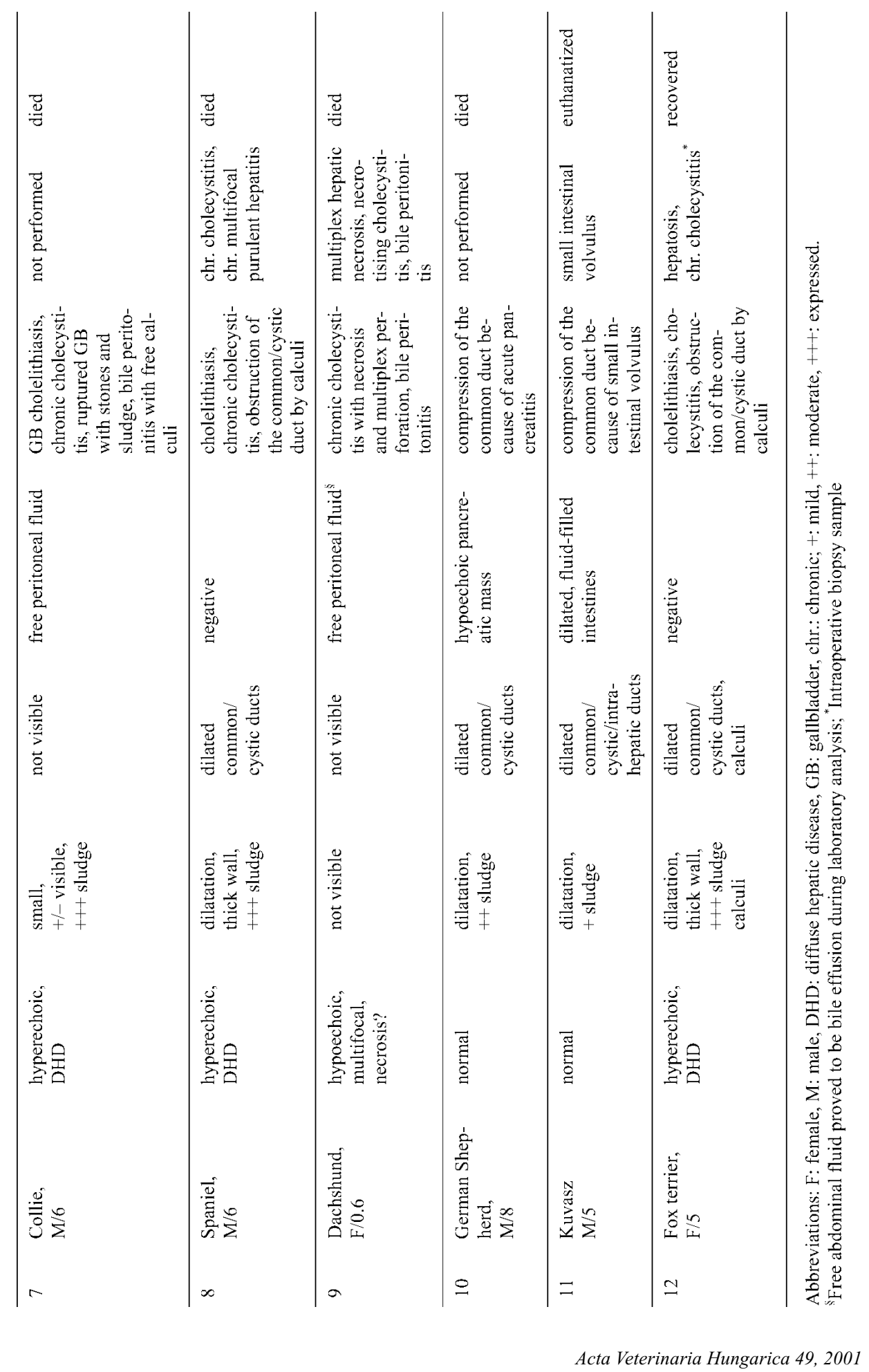


Dilatation of the intrahepatic biliary ducts (Fig. 3) together with gallbladder distension and dilatation of the extrahepatic ducts were seen in 2 dogs. Gallbladder calculi and obstructive cholelithiasis of the extrahepatic bile ducts were diagnosed ultrasonographically and were confirmed surgically in one of these patients. The other dog with dilatation of the intrahepatic ducts was the one with small intestinal volvulus. Peritoneal effusion was detected in four dogs followed by abdominocentesis and this finding was also in correlation with laparotomy results.

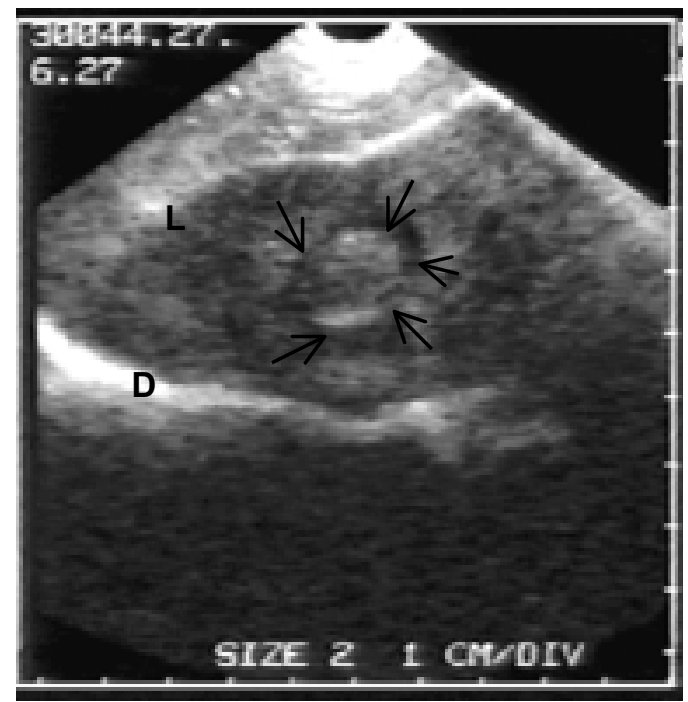

Fig. 3. Sagittal ultrasonographic image of a small, poorly delineated gallbladder filled with highly echogenic sludge in a dog (Case 7). The gallbladder is outlined by small arrows. Cholelithiasis and cholecystitis with ruptured gallbladder and bile peritonitis were diagnosed during explorative laparotomy. D: diaphragm, L: liver

Explorative laparotomy revealed various disorders causing obstruction of the gallbladder and/or extrahepatic biliary tract, as well as rupture of the gallbladder with bile peritonitis as listed in Table 1. Surgical findings confirmed the necessity of explorative laparotomy in all of the 12 dogs, but they did not correlate in all respects with the ultrasonographic results in each case. Results of indications for explorative laparotomy based on ultrasonographic findings and abdominocentesis are shown in Table 2.

When comparing ultrasound and surgical findings, gallbladder obstruction was diagnosed ultrasonographically in four dogs (Cases 2, 5, 6 and 8) which proved to be accurate during surgery but calculi were not seen in Case 8 . Thickened, mucoid bile was incorrectly interpreted as intraluminal gallbladder neoplasm by ultrasound in Case 6 . 
Gallbladder and extrahepatic biliary tract obstruction caused by choleliths was correctly diagnosed ultrasonographically in Cases 4 and 12. Extrahepatic biliary tract obstruction because of compression of the common duct was diagnosed during abdominal sonography in Cases 10 and 11 and was confirmed during laparotomy.

Peritoneal effusion due to bile peritonitis was diagnosed by ultrasonography and abdominocentesis in Cases 1, 3, 7 and 9 and these findings were in correlation with the intraoperative results.

Table 2

Comparison of ultrasonographic and laparotomy findings of 12 dogs with obstructive gallbladder and extrahepatic biliary tract diseases

\begin{tabular}{|c|c|c|c|}
\hline Ultrasonographic findings & $\begin{array}{l}\text { Number } \\
\text { of cases }\end{array}$ & $\begin{array}{l}\text { Correct/incorrect } \\
\text { ultrasound-diagnoses } \\
\text { according to laparot- } \\
\text { omy findings }\end{array}$ & Case No.* \\
\hline GB obstruction caused by bile sludge & 2 & $1 / 2$ & Cases 2,8 \\
\hline GB obstruction caused by neoplasm & 1 & $0 / 1$ & Case 6 \\
\hline GB obstruction caused by calculi & 1 & $1 / 1$ & Case 5 \\
\hline $\begin{array}{l}\text { GB and extrahepatic biliary tract obstruction } \\
\text { caused by calculi }\end{array}$ & 2 & $2 / 2$ & Cases 4,12 \\
\hline $\begin{array}{l}\text { Extrahepatic biliary tract obstruction caused } \\
\text { by pancreatic mass }\end{array}$ & 1 & $1 / 1$ & Case 10 \\
\hline $\begin{array}{l}\text { Extrahepatic biliary tract obstruction caused } \\
\text { by small intestinal ileus }\end{array}$ & 1 & $1 / 1$ & Case 11 \\
\hline Peritoneal effusion (bile peritonitis + ) & 4 & $4 / 4$ & Cases $1,3,7,9$ \\
\hline Total number of cases & 12 & $10 / 12$ & \\
\hline
\end{tabular}

GB: gallbladder; *: See detailed list of cases in Table $1 ;+$ : Based on the results of abdominocentesis

Explorative abdominal surgery performed in the 12 dogs revealed the causes of biliary obstruction in all cases. Cholecystectomy was done because of obstruction caused by inspissated bile and/or calculi of the gallbladder or extrahepatic biliary ducts in $6 \mathrm{dogs}$, and in one patient with thickened gallbladder sludge and common duct compression caused by the enlarged, oedematous pancreas. One dog with extrahepatic biliary tract compression was euthanised because of inoperable small intestinal volvulus. In 4 dogs, the ruptured gallbladder was removed via cholecystectomy and bile peritonitis was treated by intraoperative peritoneal lavage, drainage and antibiotic therapy.

Biopsy samples of the liver and gallbladder were taken during laparotomy in $4 \mathrm{dogs}$, and necropsy was performed in 5 cases. Morphological results of gallbladder alterations were in correlation with surgical findings concerning the major pathological alterations as seen in Table 1. 


\section{Discussion}

The cases of the present report were selected from our clinical material on the basis of concurrent hepatic and gallbladder ultrasonography and exploratory abdominal surgery. The advantage of the study was the possibility to correlate the ultrasonographic diagnoses with intraoperative findings and to assess the accuracy of ultrasound in the indication of surgery.

History, clinical findings and biochemical results were similar to the findings of gallbladder diseases reported previously (Schall et al., 1973; Martin et al., 1986; Kirpensteijn et al., 1993). Icterus, liver enlargement, presence of a cranial abdominal mass, or cranial abdominal fluid, as well as hyperbilirubinaemia and elevated liver enzyme activities of the serum proved to be useful indications for hepatic and gallbladder ultrasonography in accordance with literary data (Nyland and Park, 1983; Vörös, 1993). In addition, serum bile acid analysis is a useful indicator of hepatic disorders (Finn et al., 1991), but this parameter is not yet included routinely in our laboratory profile.

Abdominal radiography demonstrated liver enlargement in some patients and it was helpful in diagnosing small intestinal obstruction as a cause of common duct compression in one dog. Plain radiography can help in diagnosing gallbladder diseases only if there is gas or radiopaque calculi in the lumen of the gallbladder, in canine cholecystitis and cholelithiasis (Church and Matthiesen, 1988; Hager, 1989; Kirpensteijn et al., 1993). Although ultrasonography can be considered superior to plain radiography in diagnosing gallbladder and biliary tract diseases, radiography may be necessary for the differential diagnosis of acute diseases of other abdominal organs.

Ultrasonography is a useful technique to differentiate between hepatic and gallbladder disorders. However, gallbladder dilatation, sludge formation and intraluminal calculi are often found together with hepatic diseases (Lamb, 1990; Vörös et al., 1991; Fossum and Willard, 1995) and this tendency has also been observed in the present study. Sludge can occur secondarily to liver diseases and in anorectic dogs due to bile stasis without obstruction (Lamb, 1990; Vörös et al., 1991; Nyland et al., 1995). The amount of sludge and the grade of dilatation of the gallbladder is usually less expressed in comparison to obstructive cases. This distinction is somewhat subjective, therefore ultrasonographic gallbladder volume measurements are suggested before and after the application of cholagogue substances to test biliary patency and gallbladder function (Finn et al., 1991; Sterczer et al., 1996). However, high amount of sludge and/or choleliths can result in the obstruction of the lumen and neck of the gallbladder which indicate surgical removal (Gutbrod et al., 1987; Kirpensteijn et al., 1993). As another indicator for surgery served the presence of dilated common and cystic ducts in five dogs, one of them also demonstrating dilated intrahepatic biliary vessels. 
These findings are in accordance with experimental obstruction of the common duct (Nyland and Gillett, 1982).

Ultrasonography was accurate in detecting gallbladder obstruction in our cases, although it was not always possible to distinguish highly echogenic, inspissated bile (sludge) from calculi. Intraluminal choleliths can be diagnosed ultrasonographically if they produce acoustic shadowing and gravitation (Cartee, 1981; Nyland et al., 1995), which signs could not be observed in all of our cases with gallbladder calculi. In one dog, intraluminal neoplasm was suspected during ultrasonography, but mucoid bile accumulation was found during cholecystectomy, and this dog recovered. Gallbladder mucocele causing biliary obstruction was observed in two dogs by Newell et al. (1995). Thickening of the gallbladder wall, so-called 'double-wall appearance' due to oedema and irregular inner wall surface might be signs of cholecystitis (Nyland et al., 1995). These signs are not considered specific for cholecystitis, therefore ultrasonographically-guided percutaneous cholecystocentesis has been suggested in such cases (Rivers et al., 1997). An interesting feature, namely 'porcelain' gallbladder due to mineralisation of the gallbladder in a case of primary biliary adenocarcinoma has been described by Brömel et al. (1998a) in a dog. Ultrasound findings of this disorder included hyperechogenicity and acoustic shadowing of the thickened gallbladder wall.

Obstruction of the extrahepatic biliary tract by calculi could be diagnosed in two of our cases and intrahepatic bile duct dilatation was also seen, reflecting the longer course of the obstruction in one of these dogs. As it is known from an experimental study made by Nyland and Gillett (1982), dilatation of the gallbladder is the first change occurring after bile duct obstruction, then the extrahepatic bile ducts dilate and become tortuous in 2 to 3 days, which will be followed by intrahepatic bile duct dilatation within 5 to 7 days. Compression of the extrahepatic ducts caused by small intestinal ileus in one of our dogs and by acute pancreatitis in another one produced similar changes, in accordance with previous reports of other authors (Martin et al., 1986; Cribb et al., 1988; Diez-Bru et al., 1997).

Rupture of the gallbladder or biliary tract usually occurs as a sequel to blunt abdominal trauma, cholelithiasis with secondary cholecystitis and necrotising cholecystitis (Mullowney and Tennant, 1982; Martin et al., 1986; Parchman and Flanders, 1990). Accumulation of bile in the peritoneal cavity and bile peritonitis are serious consequences, and a delay in diagnosis and surgical treatment is directly related to a poor prognosis (Martin et al., 1986; Church and Matthiesen, 1988).

Brömel et al. (1998b) has described a canine case with cholecystitis and cholelithiasis, where the rupture of the gallbladder was diagnosed by a hypoechoic defect of the gallbladder wall during ultrasonography and by abdominocentesis. No other specific ultrasonographic signs of gallbladder and extrahepatic biliary tract rupture have been previously described to our knowledge. Of our four cases with rupture of the gallbladder, cholelithiasis with secondary cholecystitis was diagnosed surgically in 4 dogs and necrotising cholecystitis was found during lapa- 
rotomy and necropsy. Icterus was seen clinically and free abdominal fluid was detected both clinically and ultrasonographically in all four cases. Gallbladder dilatation was diagnosed by ultrasound in one of these dogs. Small, poorly demarcated gallbladder with echogenic sludge was seen in two dogs and the ruptured gallbladder could not be identified in one dog. However, direct sign of the rupture, namely defect of the gallbladder wall, could not be seen in any of our cases, contrary to a previous case report (Brömel et al., 1998b).

A small, echogenic, poorly demarcated gallbladder should be differentiated from shadowing or highly echogenic lesions within the liver, such as fibrosis, dystrophic calcification, foreign bodies, or gas (Nyland et al., 1995). According to our experiences, the presence of a small, echogenic gallbladder with sludge or calculi, or absence of the gallbladder during ultrasonography is highly indicative of gallbladder or extrahepatic biliary tract rupture if icterus and free abdominal fluid are present simultaneously. Abdominocentesis will detect bile leakage and bile peritonitis in these cases and exploratory abdominal surgery should be performed as soon as possible.

These 12 cases demonstrate the applicability of ultrasonography in the diagnosis of gallbladder and extrahepatic biliary diseases but they also reflect the limitations of distinguishing gallbladder sludge from choleliths and of the direct visualisation of gallbladder rupture.

Laparotomy was correctly indicated in all of the cases based on the following findings: (1) highly dilated gallbladder with accumulation of sludge and/or choleliths within the organ, especially at the neck of the gallbladder, (2) dilated gallbladder with dilatation of the common and cystic duct, sometimes together with dilatation of the intrahepatic biliary ducts, (3) suspicion of gallbladder rupture, based on the presence of a small echogenic gallbladder with sludge and calculi, especially together with peritoneal effusion.

Exploratory abdominal surgery and histological examination of intraoperative biopsy samples are the most reliable means of diagnosis, and surgery will provide adequate treatment in obstructive biliary diseases.

\section{Acknowledgement}

The authors thank the support received from the Hungarian Scientific Research Fund (OTKA) which provided financial means for the study in research project $\mathrm{Nr}$. 17721. 


\section{References}

Biller, D. S., Kantrowitz, B. and Miyabayashi, T. (1992): Ultrasonography of diffuse liver disease. A review. J. Vet. Int. Med. 6, 71-76.

Bjorling, D. E. (1975): Liver, biliary system and pancreas. In: Bojrab, M. J. (ed.) Current Techniques in Small Animal Surgery. 3rd edition. Lea and Febiger, Philadelphia. pp. 291-303.

Brömel, C., Smeak, D. D. and Léveillé, R. (1998a): Porcelain gallbladder associated with primary biliary adenocarcinoma in a dog. J. Am. Vet. Med. Assoc. 213, 1137-1139.

Brömel, C., Léveillé, R., Scrivani, P. V., Smeak, D. D., Podell, M. and Wagner, S. O. (1998b): Gallbladder perforation associated with cholelithiasis and cholecystitis in a dog. J. Small Anim. Pract. 39, 541-544.

Cartee, R. E. (1981): Diagnostic real-time ultrasonography of the liver of the dog and cat. J. Am. Anim. Hosp. Assoc. 17, 731-737.

Cribb, A. E., Burgener, D. C. and Reinmann, K. A. (1988): Bile duct obstruction secondary to chronic pancreatitis in seven dogs. Can. Vet. J. 29, 654-657.

Church, E. M. and Matthiesen, D. T. (1988): Surgical treatment of 23 dogs with necrotizing cholecystitis. J. Am. Anim. Hosp. Assoc. 24, 305-310.

Diez-Bru, N., Garcia-Real, I., Llorens, M. P., Toni, P., Rollan, E. and Fermin, M. L. (1997): Ultrasonographic appearance of extrahepatic biliary obstruction in the dog: a description of 7 cases. Eur. J. Comp. Anim. Med. 7, 79-83.

Finn, S. T., Park, R. D., Twedt, D. C. and Curtis, C. R. (1991): Ultrasonographic assessment of sincalide-induced canine gallbladder emptying: an aid to the diagnosis of biliary obstruction. Vet. Radiol. 32, 269-276.

Fossum, T. W. and Willard, M. D. (1995): Diseases of the gallbladder and extrahepatic biliary tract system. In: Ettinger, S. J. and Feldman, E. C. (eds) Textbook of Veterinary Internal Medicine. Diseases of the Dog and Cat. 4th edition. W. B. Saunders Co., Philadelphia. pp. 1393-1398.

Gutbrod, F., Geber, E. and Heuschmann-Fiebiger, I. (1987): Biliary tract obstruction in a dog caused by inspissated bile (in German). Prakt. Tierarzt 68, 44-45.

Hager, D. A. (1989): Diseases of the gallbladder and the extrahepatic biliary tract system. In: Ettinger, S. J. (ed.) Textbook of Veterinary Internal Medicine. Diseases of the Dog and Cat. 3rd edition. W. B. Saunders Co., Philadelphia. pp. 1555-1558.

Kirpensteijn, J., Fingland, R. B., Ulrich, T., Sikkema, D. A. and Allen, S. W. (1993): Cholelithiasis in dogs: 29 cases (1980-1990). J. Am. Vet. Med. Assoc. 202, 1137-1142.

Lamb, C. R. (1990): Abdominal ultrasonography in small animals: examination of the liver, spleen and pancreas. J. Small Anim. Pract. 31, 5-14.

Lamb, C. R. (1995): Abdominal ultrasonography in small animals. In: Goddard, P. J. (ed.) Veterinary Ultrasonography. CAB International, Wallingford. pp. 21-54.

Martin, R. A., MacCoy, D. M. and Harvey, H. J. (1986): Surgical management of extrahepatic biliary tract disease: a report of eleven cases. J. Am. Anim. Hosp. Assoc. 22, 301-307.

Mullowney, P. C. and Tennant, B. C. (1982): Choledocholithiasis in the dog: a review and a report of a case with rupture of the common bile duct. J. Small Anim. Pract. 23, 631-638.

Newell, S. M., Selcer, B. A., Mahaffey, M. B., Gray, M. L. Jameson, P. H., Cornelius, L. M. and Downs, M. O. (1995): Gallbladder mucocele causing biliary obstruction in two dogs: ultrasonographic, scintigraphic, and pathological findings. J. Am. Anim. Hosp. Assoc. 31, 467-472.

Nyland, T. G. and Gillett, N. A. (1982): Sonographic evaluation of experimental bile duct ligation in the dog. Vet. Radiol. 23, 252-260.

Nyland, T. G. and Park, R. D. (1983): Hepatic ultrasonography in the dog. Vet. Radiol. 24, 74-84.

Nyland, T. G., Mattoon, J. S. and Wisner, E. (1995): Ultrasonography of the liver. In: Nyland, T. G. and Mattoon, J. S. (eds) Veterinary Diagnostic Ultrasound. W. B. Saunders Co., Philadelphia. pp. 52-73. 
Parchman, M. B. and Flanders, J. A. (1990): Extrahepatic biliary tract rupture: evaluation of the relationship between the site of rupture and the cause of rupture in 15 dogs. Cornell Vet. 80, 267-272.

Rivers, B. J., Walter, P. A., Johnston, G. R., Merkel, L. K. and Hardy, R. M. (1997): Acalculous cholecystitis in four canine cases: ultrasonographic findings and use of ultrasonographic-guided, percutaneous cholecystocentesis in diagnosis. J. Am. Anim. Hosp. Assoc. 33, $207-214$.

Schall, W. D., Chapman, W. L., Finco, D. R., Greiner, T. P., Mather, G. W., Rosin, E. and Welser, J. R. (1973): Cholelithiasis in dogs. J. Am. Vet. Med. Assoc. 163, 469-472.

Sterczer, Á., Vörös, K. and Karsai, F. (1996): Effect of cholagogues on the volume of the gallbladder of dogs. Res. Vet. Sci. 60, 44-47.

Vörös, K. (1993): Ultrasonographic diagnosis of canine abdominal diseases. Waltham Internat. Focus 3 (4), 17-24.

Vörös, K., Vrabély, T., Papp, L. Horváth, L. and Karsai, F. (1991): Correlation of ultrasonographic and pathomorphological findings in canine hepatic diseases. J. Small Anim. Pract. 32, 627-634.

Walshaw, R. (1985): Surgical diseases. Liver and biliary system. In: Slatter, D. H. (ed.) Textbook of Small Animal Surgery. W. B. Saunders Co., Philadelphia. pp. 822-824. 